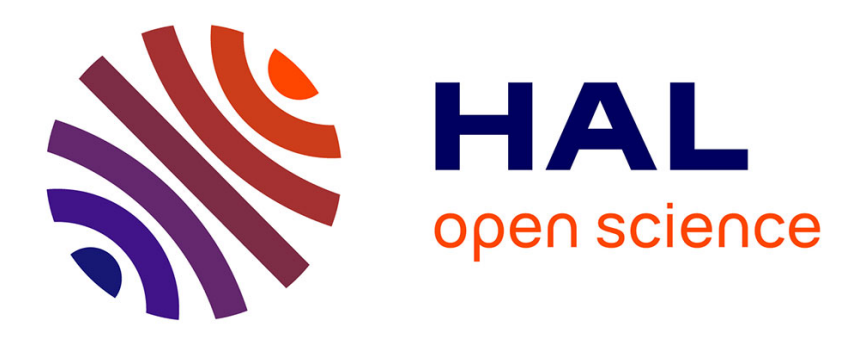

\title{
L'indépendance du Conseil constitutionnel français en question
}

Romain Espinosa

\section{To cite this version:}

Romain Espinosa. L'indépendance du Conseil constitutionnel français en question. Les Cahiers de la justice, 2015, 2015 (4), pp.547-561. 10.3917/cdlj.1504.0547 . halshs-01793450

\section{HAL Id: halshs-01793450 \\ https://shs.hal.science/halshs-01793450}

Submitted on 16 May 2018

HAL is a multi-disciplinary open access archive for the deposit and dissemination of scientific research documents, whether they are published or not. The documents may come from teaching and research institutions in France or abroad, or from public or private research centers.
L'archive ouverte pluridisciplinaire HAL, est destinée au dépôt et à la diffusion de documents scientifiques de niveau recherche, publiés ou non, émanant des établissements d'enseignement et de recherche français ou étrangers, des laboratoires publics ou privés. 


\title{
[] DOSSIER
}

\section{L'indépendance du Conseil constitutionnel français en question}

\author{
par Romain Espinosa ${ }^{1}$
}

Romain Espinosa, Doctorant contractuel en sciences économiques CRED (TEPP) à I'Université Paris II Panthéon-Assas.

Dans le sillage des travaux précurseurs menés aux États-Unis depuis une vingtaine d'années, des études européennes ont récemment mis en évidence les défauts saillants du processus décisionnel des Cours suprêmes du Portugal, de l'Espagne et de l'Italie. Un travail similaire vient d'être conduit en France sur le Conseil constitutionnel, analysant son fonctionnement et les facteurs ayant pu influencer les décisions rendues depuis 1974. II conclut que les mêmes phénomènes y sont observables: poids des considérations idéologiques et politiques lors de la prise de décision, réticences à censurer par manque d'indépendance et nécessité de repenser les mécanismes de nomination.

Following in the footsteps of ground-breaking studies that have been conducted in the United States since the twenty last years, European studies have recently pinpointed the salient failings in decision-making processes of Supreme Courts in Portugal, Spain and Italy. Similar work has been conducted in France on the Constitutional Council, analysing its workings and the factors that may have influenced the decisions it has handed down since 1974. It concludes that the same phenomena can be observed in this case, too: the weight of ideological and political considerations in decision making, a reluctance to hand down sanctions driven by a lack of independence and the need to review appointment processes.

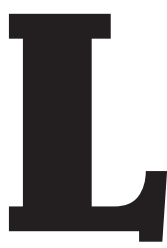

( analyse des décisions des Cours constitutionnelles a connu un essor considérable ces dernières années. À la suite des premiers travaux menés aux États-Unis à partir des années 1990, plusieurs études ont été réalisées en Europe afin de comprendre les déterminants des décisions des juges constitutionnels. Ces investigations statistiques ont permis de mettre en exergue la partialité des décisions constitutionnelles, le manque d'indépendance de ces cours et la nécessité de repenser les mécanismes de nomination des juges. Plusieurs travaux européens menés par N. Garoupa ont aussi contribué à interroger le dogme kelsenien postulant que les juges décident uniquement en droit: les juges de pays de droit civil sont autant susceptibles d'être

1. R. Espinosa, romain.espinosa@u-paris2.fr. 
influencés par leurs opinions politiques que leurs homologues américains.

Le Conseil constitutionnel ne fait pas figure d'exception et ses mécanismes de nomination y sont encore plus décriés que dans ses pays voisins. Jusqu'aux récentes réformes (2008), les nominations sans contrôle des juges au Conseil constitutionnel par les trois plus hautes personnalités de l'État - à l'exception du Premier ministre -, laissaient potentiellement la porte ouverte aux nominations les plus politisées. Plus critiquée encore, la possibilité de voir d'anciens présidents de la République siéger de droit dans une instance juridictionnelle suscite encore aujourd'hui de nombreux débats sur la question de l'indépendance du Conseil. Jusqu'alors, deux travaux menés par $\mathrm{R}$. Franck $^{2}$ ont contribué à un éclaircissement empirique de cette question. Ces derniers ont mis en lumière un taux de censure particulièrement élevé pendant les périodes de cohabitation, une influence politique de la part des conseillers n'ayant pas exercé de fonctions dans le système judiciaire et des décisions possiblement engagées en matière électorale.

Le présent article résume les récents travaux développés aux États-Unis, en Europe et en France sur les décisions des Cours constitutionnelles. Il propose ensuite un bref descriptif du mode de fonctionnement du Conseil constitutionnel français, ainsi qu'une discussion de ses décisions depuis 1974. Il s'intéresse enfin aux résultats mis en

2. R. Franck, "Judicial Independence Under a Divided Polity. A Study of the Rulings of the French Constitutional Court, 19592006 », Journal of Law, Economics and Organization, 2009, vol. $25, n^{\circ} 1$, pp. 262-284, et du même auteur, « Judicial Indepen- exergue dans le document de travail Constitutional Judicial Behavior : Exploring the Determinants of the Decisions of the French Constitutional Council ${ }^{3}$.

\section{Analyses des décisions des Cours suprêmes}

De nombreux travaux se sont intéressés aux décisions des Cours suprêmes ces dernières décennies. On constate à partir des années 1990 l'émergence d'une littérature américaine, portée par les départements de sciences politiques, qui propose une analyse empirique des décisions de justice de la Cour suprême. On observe ensuite, à partir des années 2000, la transcription des travaux américains aux systèmes européens par les départements d'économie. Suivant cette influence, deux travaux majeurs de R. Franck sont venus enrichir la compréhension du cas français.

\section{Études américaines}

Les premiers travaux empiriques traitant des décisions des Cours suprêmes ont été effectués aux États-Unis, menés de manière conjointe par les départements de sciences politiques et de statistiques. Ces travaux y ont bénéficié d'une audience considérable, principalement due à l'intérêt réel des départements de droit pour les approches quantitatives. Les principaux acteurs de cette

dence and the Validity of Controverted Elections », American Law and Economics Review, 2010, 12(2), pp. 423-461.

3. R. Espinosa, Document de travail, 2015. 
littérature ont contribué à populariser ce type d'approche (L. Epstein, W. Landes, R. Posner, A. Martin, J. Segal, K. Quinn).

Les recherches américaines se sont focalisées sur deux problématiques. D'une part, une première série d'études s'est intéressée aux préférences idéologiques des juges de la Cour suprême. En opposition aux théories formalistes, qui supposent que les juges prennent des décisions fondées uniquement en droit, ces travaux partent des prédictions développées par les théories attitudinales, qui considèrent au contraire que les juges sont motivés par un ensemble de préférences idéologiques qui déterminent leurs décisions. Cette littérature a cherché à classer les juges selon leurs préférences idéologiques. Pour cela, l'objectif a été de construire un axe sur lequel les juges figurent dans un ordre précis : plus ils se situent à gauche sur l'axe, plus ils apparaissent comme libéraux; plus ils se situent à droite sur cet axe, plus ils sont perçus comme conservateurs. On obtient donc une représentation spatiale des préférences idéologiques de chaque juge. L'intérêt de cette approche est de faire apparaître le juge médian ${ }^{4}$, considéré comme le juge pivot dans la mesure où il détermine la majorité de la cour, et donc la décision finale. Les débats techniques ont alors essentiellement portés sur la construction de l'axe et la détermination du positionnement de chaque juge. Différentes méthodes statistiques appliquées aux

4. Le juge médian correspond au juge ayant autant de juges sur sa gauche (plus libéraux) que sur sa droite (plus conservateurs). Dans le modèle américain, la cour étant composée de 9 juges, c'est le cinquième juge le plus libéral, ou, inversement, le cinquième juge le plus conservateur. décisions rendues par la Cour suprême ont été utilisées pour obtenir ce type d'axe et le classement des juges qu'il implique. Ces

\section{"L'intérêt de cette approche est de faire apparaître le juge médian, considéré comme le juge pivot dans la mesure où il détermine la majorité de la cour, et donc la décision finale. "}

approches n'aboutissent pas toujours au même type de classement, faisant ainsi changer l'identité du juge pivot.

Les universitaires américains se sont en outre intéressés à la propension individuelle des juges de la Cour suprême à censurer des dispositions. Deux articles influents se sont focalisés sur la question de l'activisme judiciaire de la part de la Cour suprême. L'objectif de ces études a été de comprendre dans quelle mesure les juges font preuve de « retenue judiciaire» (judicial self-restraint), i.e. une déférence vis-à-vis des décisions du pouvoir démocratiquement élu. D'une part, L. Epstein et A. Martin ont étudié l'ensemble des décisions de la Cour suprême de 1969 à $2009^{5}$. Leur étude a montré que les juges conservateurs (respectivement libéraux) faisaient preuve d'une plus grande retenue pour les lois conservatrices (resp. libérales). La probabilité de censure d'une loi conservatrice sur cette période était de $27 \%$ pour les juges conservateurs contre $76 \%$ pour les juges démocrates. A contrario, la probabilité de censurer une loi libérale était de 17 \% pour

5. L. Epstein, A. D. Martin, «Is the Roberts Court Especially Activist? A Study of Invalidating (and Upholding) Federal State, and Local Laws», Emory Law Journal, 2012, vol. 61, pp. 737758. 
les libéraux et de $46 \%$ pour les juges conservateurs. L'analyse statistique de ces décisions montre également que l'effet idéologique reste le plus à même d'expliquer une décision de la Cour, et ce même en prenant en compte d'autres facteurs tels que l'environnement politique, les caractéristiques des lois concernées ou le processus de sélection des lois abordées. Dans une seconde étude, L. Epstein et W. Landes se sont interrogés sur l'existence même d'une telle «retenue judiciaire ${ }^{6}$. Pour ce faire, ils ont étudié un total de 647 décisions de la Cour suprême dans lesquelles des lois fédérales étaient contestées (1937-2009). Cette seconde étude confirme les résultats précédents et conclut que le marquage idéologique est si fort qu'il en vient à remettre en cause l'existence même d'une «retenue judiciaire». L'étude affirme néanmoins que la politisation de la Cour a évolué au cours du temps : sur les 12 juges nommés avant 1952 et considérés dans l'étude, seuls 5 pouvaient être qualifiés d'idéologues, contre 14 des 18 juges nommés après 1953.

\section{Études européennes}

Plusieurs études européennes ont eu pour objectif de transposer les débats américains aux Cours constitutionnelles continentales. Ces travaux ont été principalement menés sous l'égide de N. Garoupa qui, avec ses coauteurs, a analysé les décisions des Cours espagnole, portugaise et italienne.

6. L. Epstein, W. M. Landes, «Was There Ever Such a Thing as Judicial Self-Restraint? », California Law Review, 2012, vol. 100, pp. 557-578.

7. S. Amaral-Garcia, N. Garoupa, V. Grembi, « Judicial Independence and Party Politics in the Kelsenian Constitutional Courts:
Dans un premier article, S. AmaralGarcia, N. Garoupa et V. Grembi ont analysé les décisions de la Cour constitutionnelle portugaise ${ }^{7}$. Leurs travaux ont eu pour objectif de comprendre la pertinence des débats américains dans les systèmes de droit civil. Les auteurs notent en effet que, «dans le modèle continental, les Cours constitutionnelles sont faites pour être plus conservatrices en interprétant les constitutions et les possibilités d'activisme judiciaires y sont très limitées ${ }^{8}$. Contrairement au système américain où le processus de nomination repose sur l'initiative du Président - sous le contrôle du Sénat -, la nomination des juges portugais s'effectue par le Parlement à la majorité des deux tiers pour dix d'entre eux, tandis que trois autres juges sont élus par leurs pairs. Similairement au débat libéral-conservateur, S. Amaral-Garcia et ses co-auteurs s'interrogent sur la division droite-gauche (6 des juges élus par le Parlement étant marqués à gauche et 6 marqués à droite). Leur étude portant sur les cas traités entre 1983 et 2007 conclut que les juges portugais sont eux aussi sujets à un vote idéologique et/ou politique, tout en contrôlant pour les caractéristiques des lois étudiées par la Cour. En d'autres termes, les juges nommés par la gauche (respectivement la droite) sont moins susceptibles de voter contre des lois de gauche (resp. de droite) comparativement aux lois votées par la droite (resp. par la gauche).

The Case of Portugal », Journal of Empirical Legal Studies, 2009, vol. 6, issue 2, pp. 381-404.

8. Citation originale: "In the Continental model, constitutional courts are intended to be more conservative in interpreting constitutions and are restrained from embracing active law making », Amaral et al., 2009. 
Une seconde étude, menée en Espagne, a confirmé ces résultats ${ }^{9}$. La Constitution espagnole prévoit la composition d'une Cour constitutionnelle de 12 juges. Huit d'entre eux sont élus à la majorité des trois cinquièmes par le Parlement (quatre par chambre), deux sont nommés par le Gouvernement, et deux proviennent du Conseil judiciaire. Considérant que, par leur processus de nomination, les juges siégeant à la Cour constitutionnelle ont de fortes chances d'être influencés par des motivations politiques et/ou idéologiques, N. Garoupa, F. Gomez-Pomar et V. Grembi étudient les votes individuels des juges en fonction du parti politique qui les a nommés. Cette étude conclut, ici aussi, que les juges votent en grande partie pour les intérêts des partis qui les ont nommés. Les auteurs estiment en effet que la probabilité qu'un juge vote en faveur d'une requête augmente de 39 points de pourcentage lorsque cette dernière est portée par le parti qui l'a nommée. L'étude souligne néanmoins que ce résultat est vérifié pour les questions soulevées par les partis au niveau national et non au niveau local.

La Cour constitutionnelle de la République italienne a aussi fait l'objet d'une récente étude par $\mathrm{N}$. Garoupa et $\mathrm{V}$. Grembi ${ }^{10}$. Celle-ci s'est principalement intéressée aux effets du changement institutionnel du système politique italien de 1992 qui, selon les auteurs, a transformé ce dernier,

9. N. Garoupa, F. Gomez-Pomar, V. Grembi, « Judging under Political Pressure: An Empirical Analysis of Constitutional Review Voting in the Spanish Constitutional Court ", Journal of Law, Economics, and Organization, 2011, vol. 29, issue 3, pp. 513534. passant d'une démocratie consensuelle avant 1992 à une démocratie majoritaire (à partir de 1994) ${ }^{11}$. Les auteurs se fondent sur les théories développées par Lijphart, qui oppose deux modèles de démocratie. Il définit, d'une part, le modèle consensuel dans lequel la polarisation politique est faible et qui fonctionne principalement sur la négociation et le compromis. Il décrit le modèle de démocratie majoritaire comme un système dans lequel les problèmes politiques sont tranchés par la majorité et caractérisé par un fort affrontement entre la majorité et l'opposition. L'étude de N. Garoupa et V. Grembi est la plus proche des travaux français, dans la mesure où les votes individuels des juges ne sont pas publiés. La Cour constitutionnelle de la République est composée de 15 juges, nommés en proportion égale par le président de la République, le Parlement et des représentants du pouvoir judiciaire. L'hypothèse de travail considère que les juges sont soumis à une pression moins forte dans un système parlementaire, que les auteurs assimilent à un système consensuel, dans la mesure où la majorité parlementaire est plus fragile. En revanche, les auteurs étudient la possibilité qu'un système majoritaire accentue la concentration du pouvoir (démocratie majoritaire), augmentant les risques de pression auprès des juges et amenant, de fait, à des jugements politisés. L'étude conclut que la proportion de juges nommés par des partis

10. N. Garoupa, V. Grembi, Judicial Review and Political Bias: Moving from Consensual to Majoritarian Voting, Document de travail, 2013.

11. Les auteurs définissent le régime transitoire (1992-1993) comme un gouvernement technocrate. 
Dossier L'indépendance du Conseil constitutionnel français en question

identiques au requérant augmente la probabilité de censure, pour les lois contestées après 1994. A contrario, l'analyse statistique ne permet pas d'affirmer qu'un effet similaire ait eu lieu pour les lois portées avant 1992.

\section{Études du Conseil constitutionnel français}

Deux récents travaux menés par Raphaël Franck ont ouvert l'étude quantitative des décisions du Conseil constitutionnel français.

Dans une première étude publiée en $2009^{12}$, Franck propose une analyse statistique des « décisions constitutionnelles » rendues de 1956 à 2006. Partant du constat que le Conseil constitutionnel est caractérisé par un manque d'indépendance, du moins formel ${ }^{13}$, notamment par son processus de nomination, R. Franck tente de mettre en exergue les déterminants des décisions du Conseil constitutionnel. Il s'intéresse essentiellement à deux dimensions: les périodes de cohabitation et la composition du Conseil. L'auteur avance, d'une part, que, lors des périodes de cohabitation, le Gouvernement est moins susceptible d'influencer les décisions du Conseil. Selon l'auteur, le Gouvernement et le président de la République étant de sensibilités politiques différentes, la pression exercée sur le Conseil serait plus faible. D'autre part, Franck analyse l'impact de la composition du Conseil sur ses déci-

12. R. Franck, Judicial Independence Under a Divided Polity..., op. cit.

13. R. Franck affirme: "Actually, since all the CC judges are nominated by politicians and since some $C C$ judges are even former politicians, it is likely that the decisions of the CC to fully or partially rescind laws may be explained by the political views sions. L'auteur suggère que la proportion de juges nommés par la droite (respectivement par la gauche) peut diminuer le risque de censure pour les lois portées par les partis de droite (respectivement de gauche). Franck postule aussi que la fonction préalablement exercée par les conseillers peut avoir un impact sur la décision finale: les juges ayant exercé des fonctions politiques seraient plus susceptibles de voter de manière politique que les juges ayant une formation juridique. Cette première étude conclut que les périodes de cohabitation sont associées à de plus forts taux de censure, surtout pour la législature 1997-2002, ce que Franck interprète comme un signe de la faiblesse de la gauche plurielle face au Conseil constitutionnel, assurant à celui-ci une plus grande marge de manœuvre pour rendre ses décisions. Elle apporte aussi quelques éléments suggérant que la proportion de juges nommés par la droite augmente le risque de censure pour les majorités de gauche.

Une seconde étude proposée par Franck en 2010 s'intéresse aux décisions du Conseil constitutionnel en matière électorale ${ }^{14}$. Plus précisément, ces travaux analysent 2229 décisions portant sur la validité d'élections parlementaires. De manière similaire, l'article s'intéresse à la composition du Conseil ainsi qu'aux périodes de cohabitation pour expliquer les décisions en matière électorale. En outre, l'article analyse l'impact du différentiel du

of the judges and by the political circumstances which prevailed at the time of the rulings $»$.

14. R. Franck, "Judicial Independence and the Validity of Controverted Elections ", American Law and Economics Review, 2010, 12(2), pp. 423-461. 
nombre de voix lors de l'élection contestée sur les décisions du Conseil, ce dernier étant supposé trancher les cas de fraude selon le principe de minimis non curat praetor ${ }^{15}$. Ces travaux, bien que peu détaillés d'un point de vue méthodologique, concluent sur plusieurs résultats intéressants. Premièrement, l'analyse statistique suggère que le différentiel de voix entre les candidats n'influence pas la décision du Conseil. Ce résultat est surprenant dans la mesure où le Conseil fonde son argumentation sur le principe de minimis non curat praetor. Deuxièmement, Franck montre que les juges ne semblent pas favoriser les partis qui les ont nommés. Troisièmement, cette étude affirme que les juges décident de manière indépendante du Gouvernement, à savoir qu'ils ne favorisent pas les candidats de la majorité siégeant à l'Assemblée nationale et que les périodes de cohabitation n'influencent pas leurs décisions. Quatrièmement, Franck conclut cependant que les candidats d'extrême droite ont, toutes choses égales par ailleurs, une plus grande chance de voir leur élection invalidée par le Conseil. Étant donné que l'analyse statistique de Franck prend en considération les caractéristiques de chaque élection, ce résultat semble indiquer un traitement différencié pour les candidats d'extrême droite. Ce résultat est à considérer avec prudence, dans la mesure où les élections de candidats d'extrême droite peuvent présenter des caractéristiques inobservées qui n'ont pas été prises en compte dans l'analyse statistique (effet de sélection).

15. Le principe de minimis non curat praetor stipule que les fraudes électorales ne doivent être considérées que dans la mesure où elles ont pu affecter le résultat de l'élection.

\section{Description du Conseil constitutionnel français et de ses décisions}

Le Conseil constitutionnel français a longtemps fait figure d'exception parmi les Cours constitutionnelles, mais les réformes successives de 1974 et 2008 ont fortement contribué à sa modernisation. Les décisions rendues par le Conseil constitutionnel en matière de «décisions constitutionnelles" ont connu de grandes variations depuis 1974.

\section{Description du Conseil constitutionnel}

La défiance du pouvoir politique français envers le contrôle de constitutionalité, ainsi qu'en témoigne le titre de Conseil et non celui de Cour, a longtemps entravé la création d'une Cour pouvant censurer les lois votées par le Parlement démocratiquement élu. Cette aversion s'est cependant atténuée par l'introduction, en 1946, du Comité constitutionnel, aux pouvoirs et aux possibilités de saisine néanmoins très limités. Créé en 1958, le Conseil constitutionnel s'est vu donner la possibilité de contrôler la constitutionalité des textes législatifs.

Le Conseil constitutionnel se compose actuellement de neuf juges nommés (art. 56 de la Constitution). Tous les trois ans, le président de la République, le président de 
l'Assemblée nationale ainsi que le président du Sénat nomment un conseiller, chacun pour une durée de neuf ans ${ }^{16}$. Les juges ainsi nommés ne peuvent être reconduits dans leurs fonctions. En cas d'empêchement intervenant avant la fin du mandat, les juges peuvent être remplacés pour la durée restante du mandat par un nouveau juge nommé par l'autorité ayant nommé le juge démissionnaire. Un juge ainsi nommé peut être reconduit dans ses fonctions pour la durée d'un mandat complet. Depuis la réforme de 2008, les nominations du président de la République peuvent être bloquées par une majorité des trois cinquièmes du Parlement, selon la procédure de l'article 13 de la Constitution. À ces neuf juges nommés par les plus hauts élus de la République s'ajoutent les anciens présidents de la République. Ces derniers siègent de droit au Conseil constitutionnel, pour tous les types de décisions.

Le Conseil constitutionnel est aujourd'hui amené à se prononcer principalement sur deux types de cas: les décisions constitutionnelles, d'une part, et les questions prioritaires de constitutionnalité, d'autre part. Les décisions constitutionnelles concernent les lois, traités ou règlements qui ont été soumis au Conseil constitutionnel avant leur promulgation par le président de la République. Il s'agit d'un contrôle de constitutionnalité ex ante dans la mesure où les règles concernées ne sont pas encore entrées en

16. Depuis la réforme de 2008, les nominations au Conseil constitutionnel peuvent être bloquées par une majorité des trois cinquièmes des chambres législatives (les deux chambres application. Les questions prioritaires de constitutionnalité, introduites par la réforme de 2008, concernent quant à elles les contrôles de constitutionnalité de lois déjà en application, dont la constitutionnalité a été remise en cause par un requérant lors d'un procès. Ces contrôles de constitutionnalité sont donc effectués ex post.

Le rôle du Conseil constitutionnel s'est cantonné à des décisions marginales entre 1958 et 1974. Deux facteurs contribuent à expliquer ce phénomène. D'une part, le rôle prépondérant du général de Gaulle a souvent été mis en avant pour expliquer le manque d'indépendance judiciaire: les juges siégeant au Conseil lui étaient acquis. D'autre part, les possibilités de saisine du Conseil constitutionnel sont restées très limitées jusqu'en 1974, date à partir de laquelle les députés et sénateurs se sont vus octroyer la possibilité de contester la constitutionnalité des textes votés par leur chambre. Actuellement, le Conseil constitutionnel peut être saisi, en matière de décisions constitutionnelles, par six autorités : le président de la République, le président du Sénat, le président de l'Assemblée nationale, le Premier ministre, 60 députés et/ou 60 sénateurs. Le Conseil ainsi saisi doit rendre une décision dans un délai d'un mois à compter de la saisine (art. 61 de la Constitution).

pour les nominations du président de la République et leur chambre respective pour les présidents du Sénat et de l'Assemblée nationale). 


\section{Description des cas traités par le Conseil constitutionnel depuis 1974}

L'article Constitutional Judicial Behavior: Exploring the Determinants of the Decisions of the French Constitutional Council ${ }^{17}$ repose sur une base de données qui comporte la quasitotalité des décisions constitutionnelles rendues par le Conseil constitutionnel depuis 1974.

L'activité du Conseil a considérablement augmenté au cours des années. En effet, alors que le Conseil a rendu moins de 40 décisions constitutionnelles pendant les quatre dernières années de la Ve législature (19741978), il a été amené à se prononcer un peu moins de 100 fois sous la présidence de Nicolas Sarkozy (v. Graph. 1). On observe de plus que la saisine parlementaire est devenue la première source de demande de contrôle de constitutionnalité (v. Graph. 2). L'introduction du Conseil constitutionnel comme étape du processus législatif - et donc politique - apparaît en effet dès 1974-1978, période à laquelle presque la moitié des saisines est d'origine parlementaire. Ce phénomène s'est renforcé puis stabilisé dès la VIe législature (1978-1981). La proportion de décisions constitutionnelles demandées par les parlementaires oscille depuis entre $60 \%$ et $85 \%$ du nombre total de décisions constitutionnelles.

17. R. Espinosa, Analyse Économique de la Norme Juridique. des Origines Constitutionnelles à la Mise en Euvre par le juge, 2015 (Thèse de Doctorat).
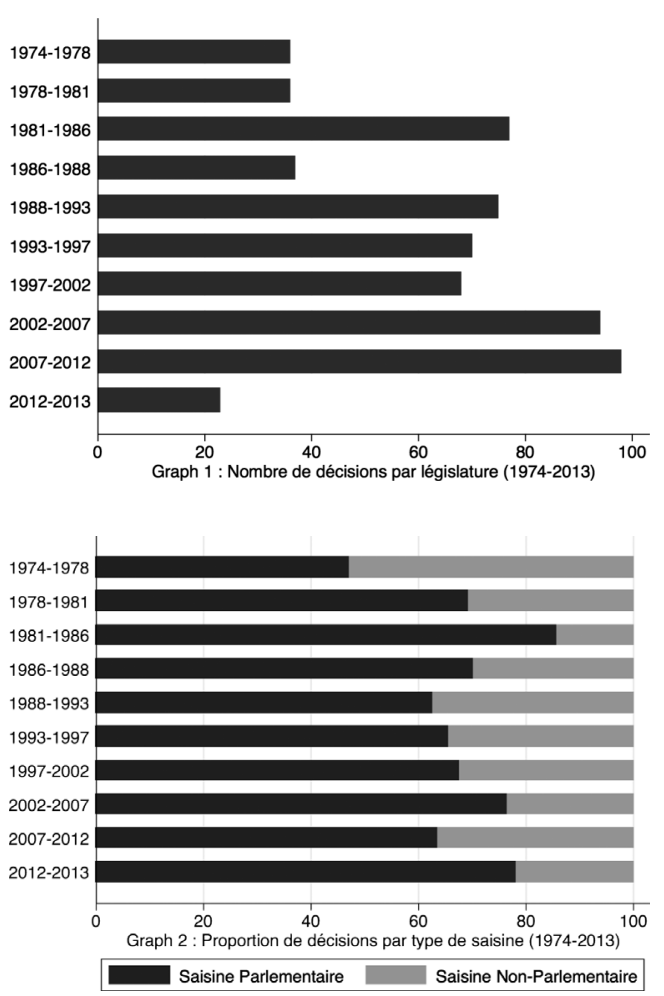

\section{Trois théories sur le processus décisionnel du Conseil constitutionnel}

La présente section discute des résultats empiriques mis en évidence par l'article Constitutional Judicial Behavior: Exploring the Determinants of the Decisions of the French Constitutional Council. Dans la continuité des travaux cités précédemment, cette étude s'intéresse à l'indépendance du Conseil 
Dossier L'indépendance du Conseil constitutionnel français en question

constitutionnel au travers de trois théories. La première théorie analysée dans le papier reprend le modèle américain ainsi que les travaux de Franck sur le Conseil constitutionnel. Il s'agit du modèle attitudinal qui s'intéresse aux décisions du Conseil eu égard à sa composition (politique). La deuxième théorie reprend en partie les travaux américains sur la «retenue judiciaire» et teste empiriquement certaines prédictions formulées par D. Schnapper ${ }^{18}$. Enfin, la troisième théorie, appelée «indépendance opportuniste », analyse les décisions du Conseil constitutionnel dans un cadre politique plus large, à savoir dans les périodes de cohabitation et lorsque les majorités de l'Assemblée nationale et du Sénat divergent.

La stratégie empirique mise en place dans le papier en question consiste à analyser les décisions constitutionnelles rendues entre 1974 et 2013. En d'autres termes, l'objectif est de comprendre quels facteurs influencent la décision de validité ou d'invalidité prise par le Conseil constitutionnel. Ainsi, la variable d'intérêt, notée «invalidation », est égale à 1 si une loi est censurée (en partie ou en intégralité) par le Conseil, et égale à 0 si la loi est confirmée (en partie ou en intégralité). La procédure de code de cette variable est reprise à partir de la catégorisation du Conseil qui, sur son site Internet, catégorise les décisions comme «conformité» ou «non-conformité ».

18. D. Schnapper, Une sociologue au Conseil constitutionnel, Paris, Éditions Gallimard, 2010.

19. L. P. Feld, S. Voigt, « Economic growth and judicial independence: Cross-country evidence using a new set of indicators »,

\section{Le modèle attitudinaI}

Le modèle attitudinal postule que les juges sont motivés par des considérations idéologiques et/ou politiques lorsqu'ils rendent des jugements. De manière plus générale, la littérature en économie du droit a distingué ces deux types de considérations. Les motivations idéologiques font référence à un ensemble de croyances et de préférences, caractérisant chaque juge et lui donnant une sympathie naturelle pour les arguments énoncés par un des requérants. Les motivations politiques visent, quant à elles, le jeu d'influence entre les partis et les juges, au travers de perspectives de carrière ou d'autres avantages ou sympathies pour les partis politiques. Bien que ces distinctions théoriques soient importantes, l'analyse statistique se révèle incapable de distinguer les deux phénomènes en étudiant les décisions de justice. En effet, un vote quasi systématique en faveur des arguments portés par un des requérants peut refléter aussi bien une sympathie envers ses arguments qu'une fidélité politique.

Eu égard à la littérature sur l'indépendance de l'autorité judiciaire, le Conseil constitutionnel demeure très critiquable à plusieurs égards. L. Feld et S. Voigt affirment en effet que la nomination des juges doit se tenir autant que possible à l'écart des autres branches du pouvoir afin de garantir une plus grande indépendance ${ }^{19}$. Le Conseil constitutionnel fait, de facto, défaut à cette condition

European Journal of Political Economy, 2003, vol. 19, pp. 497527. 
en donnant un pouvoir central à des acteurs éminemment politisés. La présence des anciens présidents de la République aggrave ce phénomène en introduisant dans le Conseil des personnalités engagées de premier plan. De plus, L. Feld et S. Voigt soulignent que l'indépendance du pouvoir judiciaire est garantie par la nature et la durée des mandats. À cet égard, le mandat de neuf ans est moins à même de garantir l'indépendance du système judiciaire que les mandats à vie, à l'instar des juges de la Cour suprême américaine.

Considérant ces failles dans l'indépendance du Conseil constitutionnel, les travaux sur le Conseil constitutionnel français ont eu pour objectif de tester l'influence du politique sur les décisions constitutionnelles. Pour ce faire, l'analyse empirique croise la variable «invalidation» décrite précédemment avec deux dimensions politiques: $1^{\circ}$. La proportion de juges nommés par un représentant politique de droite et $2^{\circ}$. Le fait que le président du Conseil constitutionnel ait été nommé par un représentant politique de droite. La première dimension se décompose en deux variables. Entre 1974 et 2013, l'étude utilise la proportion de juges nommés par la droite ayant un mandat en cours lorsque la décision a été prise (mandat_droite). Entre 1995 et 2013, il est possible de connaître

20. Le président du Conseil possède un pouvoir important dans le processus décisionnel, tant par son statut de « président » que par ses compétences (il détermine les rapporteurs des lois controversées).

21. Les variables de contrôle permettent de prendre en considération les caractéristiques propres à chacune des lois étudiées. Ici, elles contiennent des informations sur: le sujet sur lequel porte le texte de loi (loi économique, loi de finances, loi sur les l'exacte composition du Conseil pour chaque décision, à savoir quels juges ont assisté à la décision (prop_droite) ${ }^{20}$. Une seconde variable est donc créée, égale à la proportion de juges nommés par la droite présents à chaque décision précise. Concernant la seconde dimension, on crée une variable égale à 1 lorsque le président du Conseil a été nommé par la droite (pres_droite). Il convient de noter que ces variables sont définies en prenant la droite comme référentiel mais qu'elles peuvent être renversées pour être interprétées pour la gauche.

L'analyse économétrique menée propose alors d'estimer la probabilité qu'un texte soit invalidé considérant: $1^{\circ}$. Que le texte juridique étudié ait été contesté sous une législature de droite; $2^{\circ}$. La proportion de juges nommés par un représentant politique de droite (mandat_droite ou prop_droite) ; $3^{\circ}$. Le parti politique qui a nommé le président du Conseil (pres_droite); $4^{\circ}$. D'autres variables de contrôle ${ }^{21}$. Le modèle statistique inclut aussi d'autres variables d'intérêt décrites dans les sections suivantes.

Concernant le modèle attitudinal, l'objectif de l'économétrie du papier est de mesurer l'impact de l'interaction entre le fait qu'une loi soit votée par une législature de droite et le nombre de juges nommés par la droite. Ceci permet en effet de voir si les lois votées

institutions, loi électorale, loi sur les collectivités territoriales), l'autorité qui a demandé la saisine (députés seuls, sénateurs seuls, députés et sénateurs ensemble, Premier ministre, président de la République, président du Sénat, président de l'Assemblée nationale), le type de texte (loi ordinaire, loi organique, résolution/règlement, traité), des effets spécifiques à chaque législature, et le nombre de jours entre la saisine et la décision. 
Dossier L'indépendance du Conseil constitutionnel français en question

par la droite ont moins de chances d'être invalidées quand le Conseil est dominé par des juges nommés par la droite. Un coefficient de corrélation partiel «négatif » signi-

\section{« J.-Cl. Colliard fait remarquer qu'un conseiller qui voterait systématiquement en faveur des positions de celui qui l'a nommé se déshonorerait vis-à-vis de ses collègues. "}

fierait qu'on observe une censure moins fréquente pour les lois votées par la droite lorsque le Conseil est composé de juges nommés par cette majorité, ceteris paribus. L'hypothèse statistique par défaut est que cette proportion de juges nommés par la droite n'a pas d'influence sur la probabilité de censure d'une loi de droite. Le test statistique consiste à savoir si cette hypothèse est «violée » et, le cas échéant, si la corrélation associée est «positive » ou «négative».

L'article conclut que l'hypothèse de non influence est violée et que les variables politiques sont en effet corrélées avec la décision d'invalidation. L'étude empirique n'est cependant pas totalement capable d'affirmer si l'effet politique est dû au président du Conseil constitutionnel ou bien à la proportion de juges nommés par la droite, même si elle suggère que l'effet est dû au second phénomène. Elle montre cependant que, plus le Conseil est marqué à droite (c'est-à-dire composé de juges nommés par des représentants de droite), moins les lois de droite ont de chances d'être censurées. Ce résultat est bien entendu relatif et peut s'interpréter de

22. D. Schnapper, Une sociologue au Conseil constitutionnel, op. cit., p. 304. manière symétrique: plus le Conseil est marqué à gauche, plus les lois de droite ont de chances d'être censurées.

Un tel résultat est néanmoins contesté par les membres du Conseil constitutionnel, ainsi qu'en témoigne D. Schnapper: «J.-Cl. Colliard fait remarquer qu'un conseiller qui voterait systématiquement en faveur des positions de celui qui l'a nommé se déshonorerait vis-à-vis de ses collègues » 22 . Ce résultat est néanmoins cohérent avec les travaux de R. Franck, qui concluait que la proportion de juges nommés par la droite et ne bénéficiant pas d'une formation juridique influençait la probabilité de censure des lois de droite. Bien que D. Schnapper ne reconnaisse pas le vote politique ou idéologique, elle déclare néanmoins: "Toute décision constitutionnelle, même si elle est fondée sur des arguments juridiques, comporte une dimension politique ou, en tout cas, à portée politique» (p. 207); ou encore «Le vote n'est jamais uniquement politique, mais le vote selon les critères politiques (...) n'est pas non plus inconnu » (p. 305) 23 .

\section{La retenue judiciaire}

La déférence du Conseil constitutionnel envers les institutions démocratiquement élues s'est établie dès les premières heures du Conseil et a été cultivée par le système de nomination ainsi que par la pression politique exercée sur les juges. La tradition légale française, remontant jusqu'à Montesquieu, a

23. Id. 
toujours considéré le Parlement comme la (seule) représentation légitime des aspirations du peuple. L'autorité judiciaire - généralement appelée «pouvoir» judiciaire hors de l'Hexagone - a ainsi été cantonnée au rôle de simple interprète de la loi. Le Conseil constitutionnel s'inscrit dans cette tradition ${ }^{24}$ et les conseillers reconnaissent la prépondérance du Parlement, se limitant ainsi à un contrôle de constitutionalité restreint.

L'article s'intéresse à cette question de la «retenue judiciaire» et propose trois pistes d'investigation. Premièrement, il s'intéresse au lien entre probabilité d'invalidation et nombre de censures prononcées depuis le début de la session parlementaire. En effet, D. Schnapper indiquait à propos du Conseil: «Un président avançait parfois, avec humour, que le Conseil avait épuisé son 'quota' de censure pour l'année ou pour la session parlementaire en cours, qu'il ne pouvait plus se permettre d'en imposer une nouvelle» ${ }^{25}$. Un coefficient négatif associé au nombre de censures prononcées depuis le début de la session parlementaire indiquerait qu'une loi a une chance plus faible d'être invalidée lorsque beaucoup d'invalidations ont déjà été prononcées. Ceci serait une preuve d'une retenue judiciaire ou, pire encore, d'un manque d'indépendance de la part du Conseil. Deuxièmement, l'analyse économétrique considère également le nombre de censures prononcées le même jour, supposant ainsi que le Conseil puisse

24. Id., p. 49: «La tradition politique est, en France, légicentriste: c'est la loi et la volonté du peuple s'exprimant par la bouche de ses représentants qui disposent de la légitimité ». vouloir limiter son nombre de censures pour ne pas susciter de projets de réforme à son encontre. Enfin, l'étude cherche à mesurer l'impact de la décision précédente sur les décisions du Conseil: si celui-ci cherche à éviter des invalidations trop fréquentes, on peut imaginer qu'il soit plus enclin à valider une loi si sa décision précédente est une invalidation. Une corrélation négative entre la décision précédente (invalidation) et la décision actuelle refléterait ce phénomène.

L'article conclut que: $1^{\circ}$. Le Conseil est moins susceptible d'invalider une loi lorsqu'il a déjà prononcé plusieurs invalidations au cours de la session parlementaire; et $2^{\circ}$. il a moins de chances d'invalider une loi si sa dernière décision était déjà une invalidation. Le premier résultat est robuste au fait que les lois votées en fin de session parlementaire

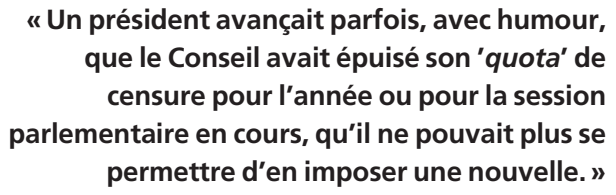

soient de qualité différente des lois votées en début de session (induisant ainsi une propension plus forte ou plus faible à être invalidées). Ces conclusions statistiques confirment l'idée que les juges sont réticents à censurer les lois votées par le Parlement. Elles interrogent notamment sur l'indépendance du Conseil : le Conseil constitutionnel

25. Id., p. 256 
Dossier L'indépendance du Conseil constitutionnel français en question

réserverait-il ses censures pour les cas les plus inconstitutionnels?

\section{Indépendance opportuniste}

La dernière dimension étudiée dans le papier concerne la théorie de «l'indépendance opportuniste», selon laquelle le Conseil constitutionnel jouit d'une plus grande indépendance lorsque les institutions politiques sont divisées. Les travaux précédents de Franck confirment cette théorie. Dans le prolongement de ces travaux, le papier considère les décisions d'invalidation dans deux cas de figure: $1^{\circ}$. Pendant les périodes de cohabitation et $2^{\circ}$. Lorsque les majorités du Sénat et de l'Assemblée nationale ne coïncident pas.

L'analyse empirique de cette dimension souffre de difficultés techniques dans la mesure où, entre 1995 et 2013, les périodes de cohabitation et de non concordance des majorités se recouvrent grandement (ce qui

\section{« Le Conseil constitutionnel français est,}

par son processus de nomination,

et par l'influence politique exercée

par les institutions élues, largement critiqué

pour son possible manque d'indépendance. "

entraîne en termes économétriques, un problème de colinéarité). Le papier conclut néanmoins que le taux de censure est plus élevé pendant les périodes de cohabitation (à savoir pendant la législature Jospin). Les résultats de l'étude ne détectent pas d'effet significatif pour les périodes d'opposition entre les chambres.

La plus grande propension à censurer pendant les périodes de cohabitation est cohé- rente avec la théorie d'indépendance opportuniste. Il convient de noter cependant que d'autres explications alternatives peuvent rendre compte de ce résultat. À titre d'exemple, il est possible que, pendant les périodes de cohabitation, le Gouvernement propose des lois moins consensuelles, forçant de fait le Conseil à censurer davantage.

\section{Conclusion}

Les analyses statistiques des décisions des Cours constitutionnelles représentent un phénomène relativement récent dans les pays de droit civil. Les travaux précurseurs menés aux États-Unis ont permis une meilleure compréhension du processus décisionnel de la Cour suprême. Des travaux similaires ont été menés en Europe et ont conclu que les mêmes phénomènes s'observaient dans les Cours constitutionnelles de droit civil. De récents travaux en France sont arrivés à des conclusions similaires.

Le Conseil constitutionnel français est, par son processus de nomination, et par l'influence politique exercée par les institutions élues, largement critiqué pour son possible manque d'indépendance. L'article Constitutional Judicial Behavior: Exploring the Determinants of the Decisions of the French Constitutional Council conclut que le Conseil souffre en effet de sérieux manques d'indépendance. Il montre, d'une part, que des considérations idéologiques et politiques sont en jeu lors de la prise de décision (couleur de l'autorité qui a nommé les juges et/ou le président du Conseil) et, d'autre part, que le Conseil se restreint fortement dans ses décisions de censure. Ces deux conclusions 
mettent en cause l'indépendance du Conseil constitutionnel.

Bien que les récentes réformes aient apporté des changements favorables à une plus grande indépendance du Conseil, avec l'introduction d'un veto parlementaire pour les nominations présidentielles, des doutes persistent quant à l'indépen- dance du Conseil. La publication d'opinions individuelles pourrait accroître la transparence du contrôle. De même, une réflexion sur un changement de statut du Conseil vers une véritable Cour constitutionnelle permettrait d'apaiser les inquiétudes soulevées par ce travail et les études précédentes. 